\title{
Review of Neurosurgical services at B\&C Hospital in last 3 years
}

\author{
Dinesh K. Thapa ${ }^{1}$ \\ ${ }^{1}$ Department of Neurosurgery, B \& C Medical College Teaching Hosptial, Birtamode, \\ Jhapa.
}

Neurosurgery was started in Far-Eastern Nepal as Department of Neurosurgery at B\&C Hospital in early 2015. Prof.Dr.Basant Pant played a key role to establish and give astounding start with all technical supports possible. Dr. Chandra P Limbu and I joined the institute at the beginning from ANIAS. Since last one year the department is led by Dr. Pankaj Raj Nepal, who is one of the finest trainee of Prof. Upendra P. Devkota.

The set up of the operating theatre is allowing us to do almost all types of neurosurgical procedures. In last three years there were 336 head injuries cases among which acute subdural hematoma was the commonest one. There were 48 cases of cervical spine injuries cases admitted over this duration. Hemorrhagic stroke requiring surgical procedure were 46 followed by 11 cases of malignant MCA infarction. Tumors were of different types and were 83 cases during this period. Aneurysms were 46 in number who underwent clipping.

Keywords: Head injury, aneurysms, hemorrhagic stroke, malignant MCA infarction

$\mathrm{N}$ eurosurgery was started in Far-

Eastern Nepal as Department of Neurosurgery at B\&C Hospital in early 2015 by signing memorandum of understanding between Annapurna Neurological Institute and Allied Sciences (ANIAS) and B\&C Medical College Teaching Hospital to provide services in door steps. The renowned senior neurosurgeon and chairman of ANIAS, Prof.Dr.Basant Pant played a key role to establish and give astounding start with all technical supports possible. Dr. Chandra P Limbu and I joined the institute at the beginning from ANIAS. Since last one year the department is led by Dr. Pankaj Raj Nepal, who is one of the finest trainee of Prof. Upendra P. Devkota (Father of Modern Neurosurgery of Nepal) ${ }^{2}$. Since its start many patients had received different kinds of neurosurgical as well neurological support. It is situated in Birtamode, rapidly growing small town of Far Eastern Nepal.

Our hospital is situated near the IndoNepal border where Siliguri and Biratnagar were the key referral centers before. Being about two hours distant from larger cities with neurosurgical facilities, most trauma patients were unable to get timely intervention. So we aimed to save patients with trauma at our center though all kinds of elective cases are in routine schedule. Neurosurgery facility was already in function on eastern part of Nepal at Biratnagar and Dharan. ${ }^{1}$ 
Hereby, I am briefly summarizing cases managed surgically in different stream of neurosciences at our center in last 3 years.

\section{Head Injury:}

The incidence of road traffic accident is high all over Nepal and south Asia, where about 7/100000 population dies in accident in 2011-2012 ${ }^{3}$. B\&C Hospital is located at the edge of East-West Highway and similar to other parts of the country this part is also prone to road traffic accident. So the incidence of trauma registry is high at our center. The diagrams below summarize the overall scenario of head injury patient who were managed surgically.

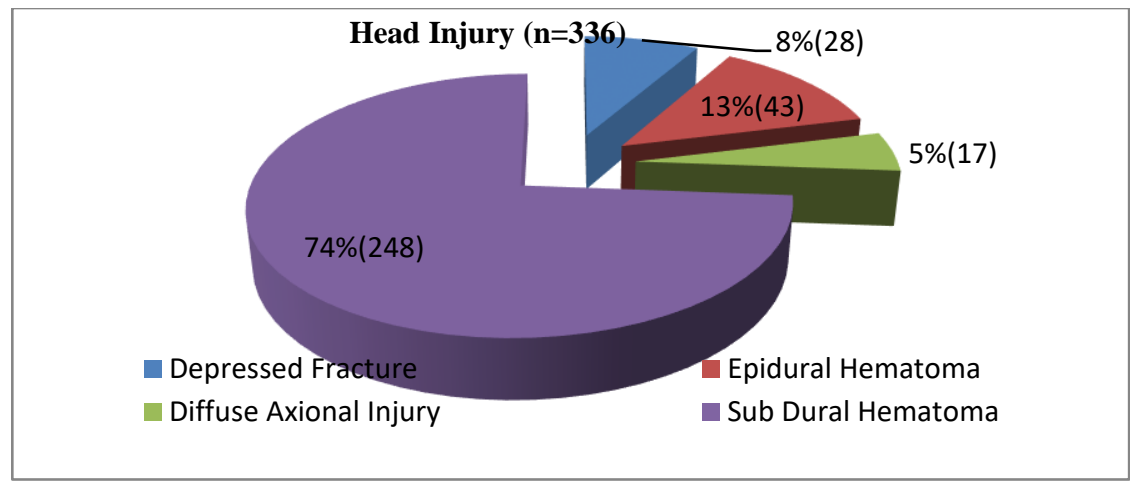

Figure 1: Overall Head injuries cases

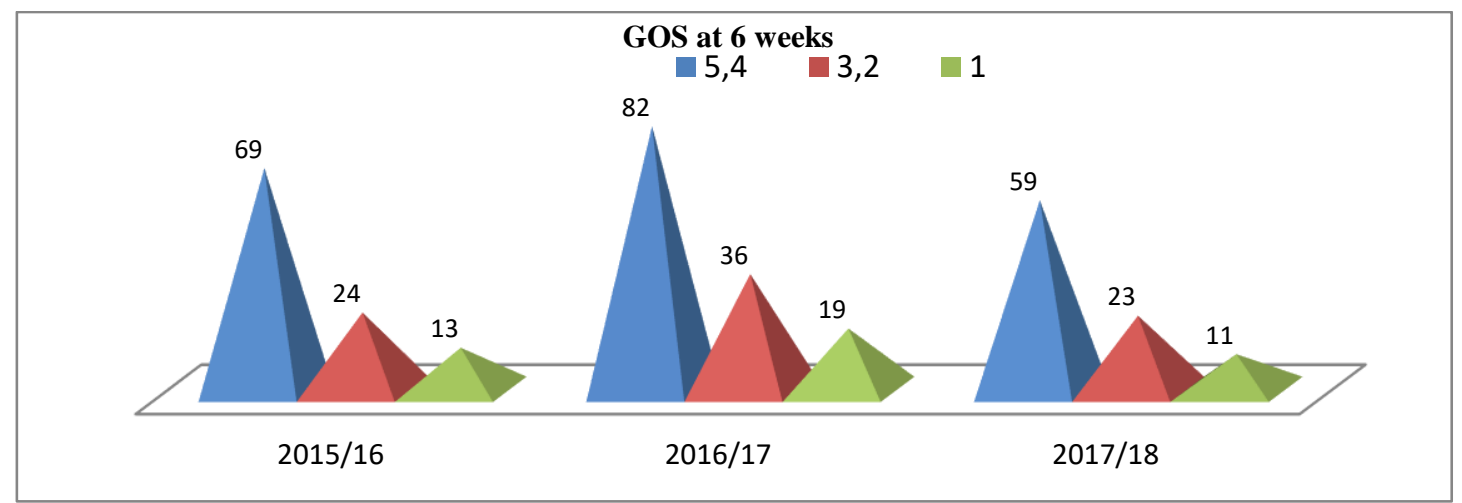

Figure 2: GOS at 6 weeks

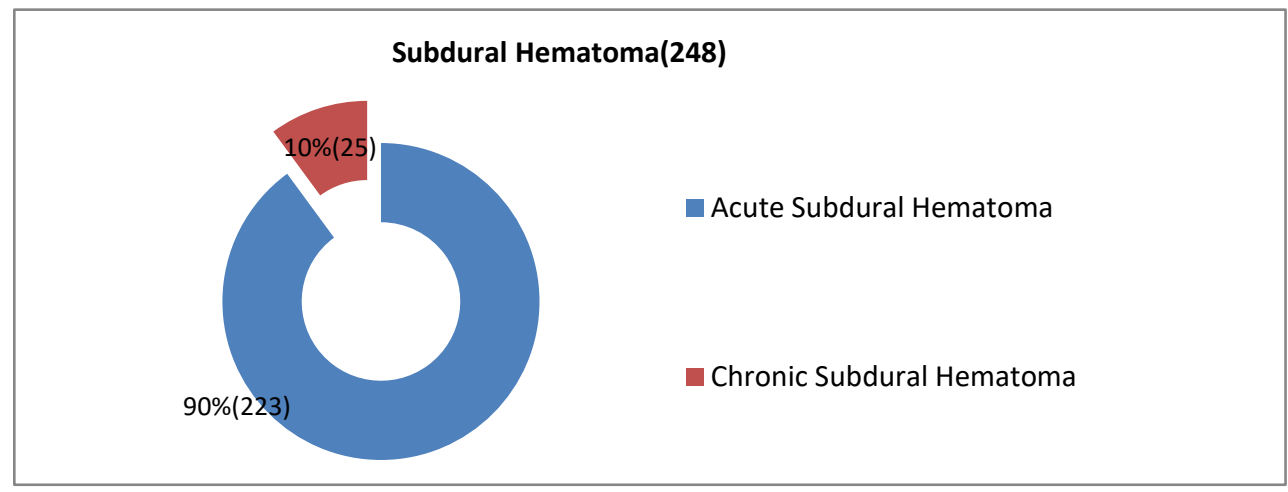

Figure 3: Overall Subdural Hematoma 


\section{Spinal Injuries:}

We receive many patients in Emergency Room with history of paresis or plegia of lower limbs or all four limbs. Most of the cases of cervical spine injuries were due to RTA followed by fall from tree or height. Most common level of subluxation was seen at C5-C6, C6-C7. We are doing anterior fixation as well as posterior fixation if needed. Most of the cervical injuries got operated were of ASIA B and C. Morbidity and mortality is high among patients presented with neurology of ASIA A.

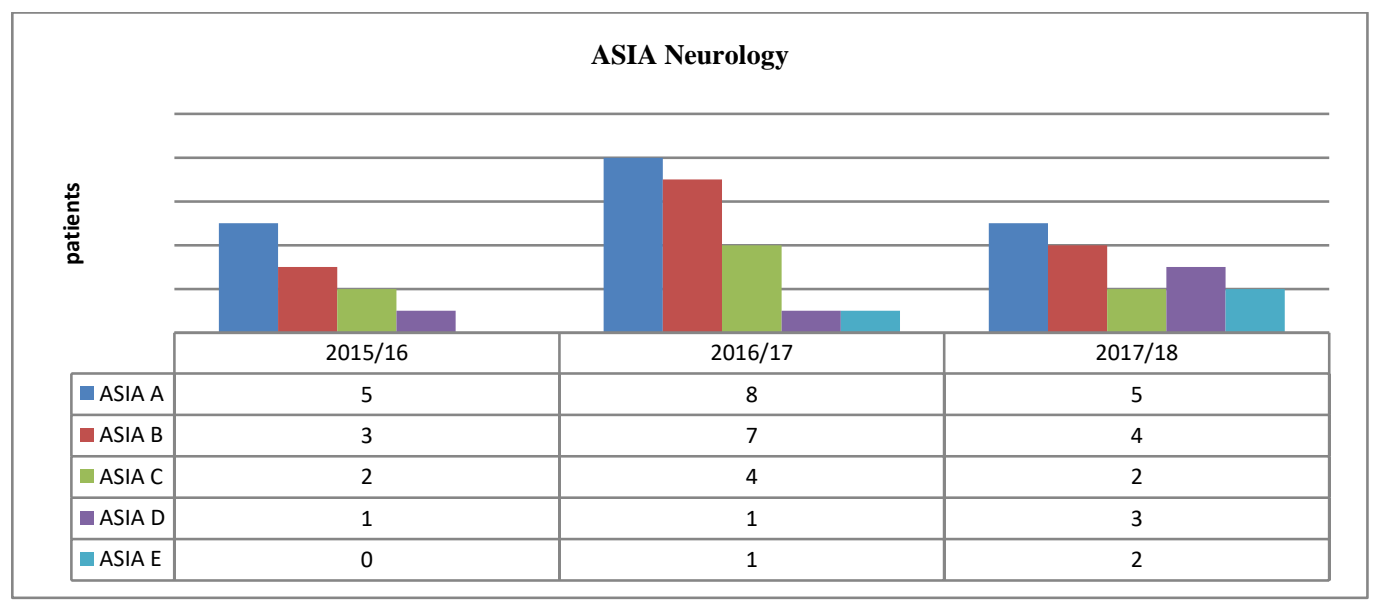

Figure 4: ASIA Neurology of Operated cases

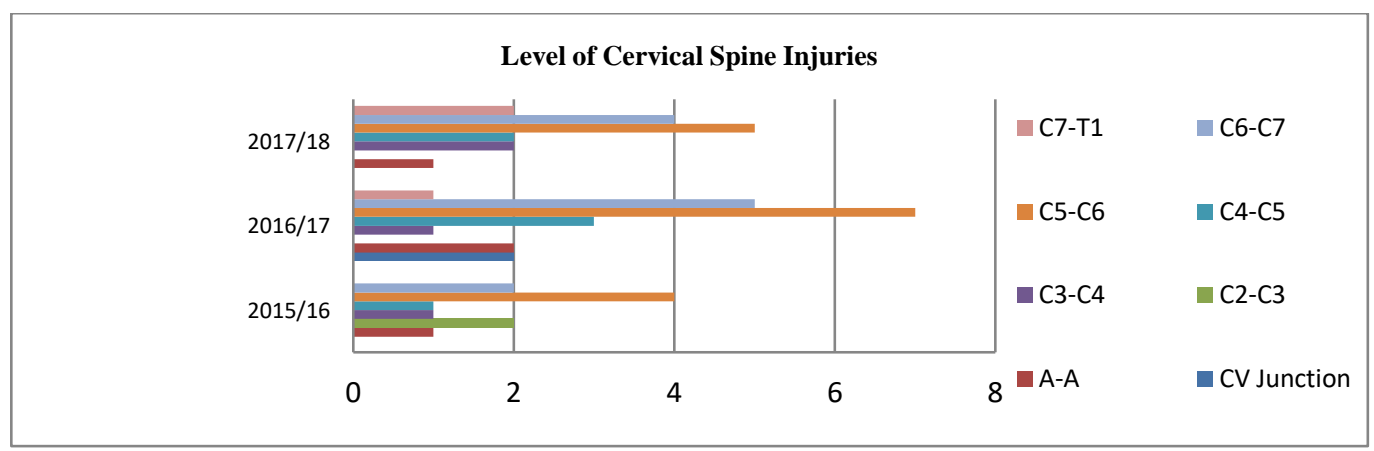

Figure 5: Frequencey of levels of cervical spine injuries

\section{Cerebrovascular Diseases(CVD):}

The incidence of CVA is in increasing trend among Nepalese due to diet modifications and lack of adequate physical mobility. We are getting increasing number of admissions and surgery done for it. The prevalence of hemorrhagic stroke (HCVA) is higher than Ischemic CVA (ICVA) at our center. Most of the patients were diagnosed case of hypertension with deferred taking medicines,

\section{4}

egneuro Volume 01, 2019 few are only associated with Diabetes or cardiac diseases as well.

Most of the patients with HCVA needing surgery were managed with craniotomy and evacuation of hematoma and few needed Decompressive Craniectomy (DC). The surgical need for ICVA in malignant MCA infarction is to protect further deterioration due to swelling. We are all set to go for IVtPA and Mechanical Thrombectomy for the possible case. There are many patients getting 
to ER with in 1 hour of weakness, so these patients will be in benefit with the service.

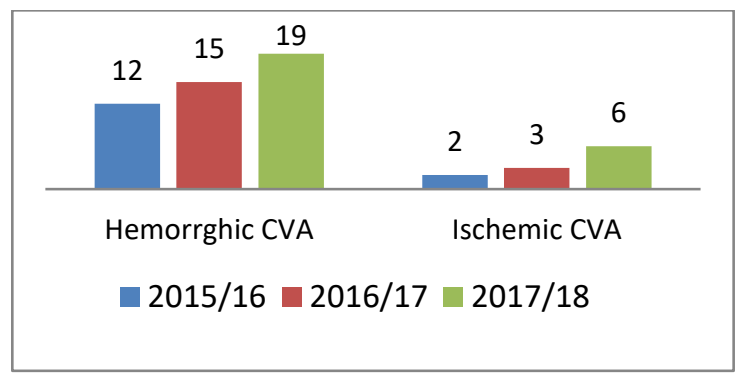

Figure 6: Frequencies of stroke who underwent surgeries.

\section{Central Nervous System Tumors:}

The numbers of elective cases were not very high due to high credibility capacity of locals and choosing centers in bigger cities and abroad. Though, we are performing all possible surgeries for different kinds of CNS pathologies

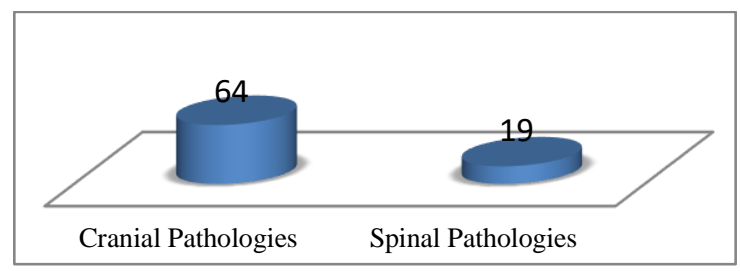

Figure 7: Distribution of tumors

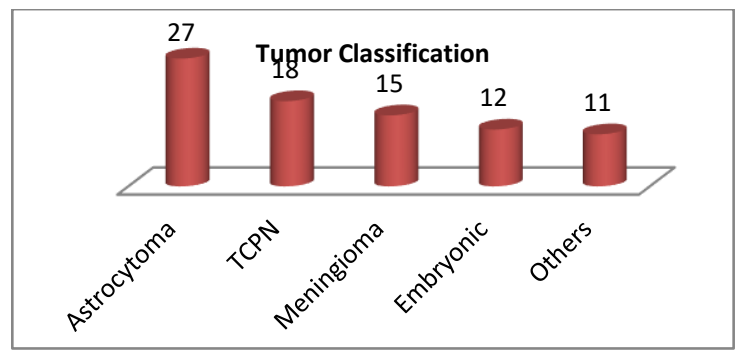

Figure 8: Different types of tumors

\section{Vascular Surgeries:}

We are routinely performing vascular surgeries for patients presenting with ruptured Aneurysm, AVMs and Cavernomas. We also encountered few cases of Dural Arterio Venous Fistula (dAVF) and Cavernoma associated with Developmental Venous Anomaly (DVA). The diagram below presents total vascular surgeries performed during the period.

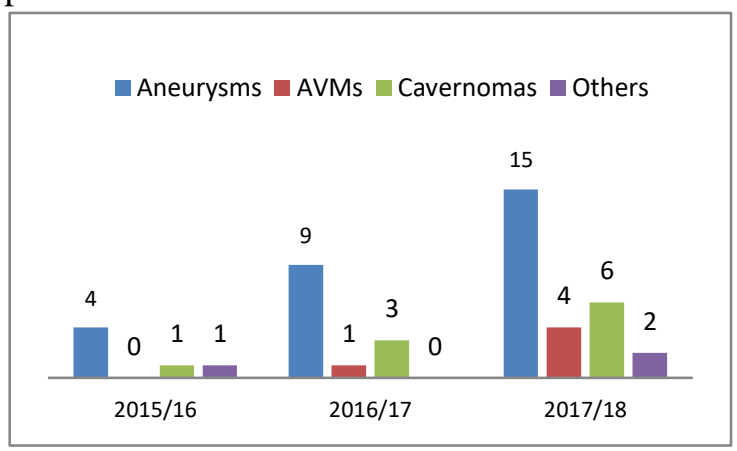

Figure 9: Distribution of Vascular operated cases.

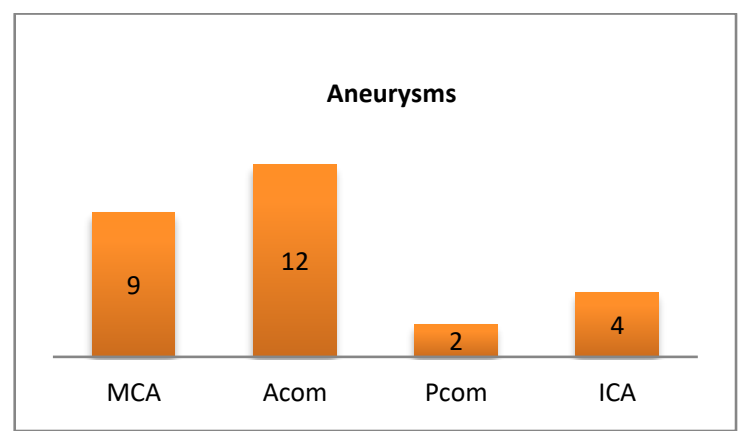

Figure 10: Distribution of Aneurysms cases.

We are here to provide all kinds of neurosurgical supports in this region aiming to minimize mortality and morbidity of patients believing in Time is Brain.

\section{References:}

1. Bhandari R, Mahato IP, Paudel M, Giri R. Head Injury - A Case Profile Study from Eastern Region of Nepal. Health Renaissance 8(2): 11-13, 2010

2. Devkota UP, Aryal KR. Result of surgery for ruptured intracranial aneurysms in Nepal. British Journal of Neurosurgery 15: 13-16, 2001. 
Thapa DK et al.

3. Karkee R, Lee AH Epidemiology of road traffic injuries in Nepal, 2001-2013: systematic review and secondary data analysis, BMJOpen 2016;6:e010757. doi: 10.1136/bmjopen2015-010757 